\title{
Interaction Diagrams for Ambient and Heated Concrete Sections
}

\author{
Angus Law ${ }^{1}$, Martin Gillie ${ }^{2}$
}

\begin{abstract}
Bending moment axial force interaction diagrams are a commonly used tool in any design office. When designing for fire conditions, the large axial forces which develop place an additional importance on the consideration of the interplay between axial forces and moments. This paper presents a new method for calculating the biaxial bending moment/axial force capacity for a general section through the use of the sectional tangent stiffness. A beam-column section subject to fire is assessed, and comparisons made with simplified design tools. It is concluded that derivation of the interaction surface from the tangent stiffness matrix is possible, and that current simplified methods for fire design cannot be assumed conservative.
\end{abstract}

\section{Keywords}

Interaction Diagrams, High Temperatures, Concrete, Tangent Stiffness, Fire, Biaxial Bending

\section{INTRODUCTION}

The design of reinforced concrete sections requires the specification of a number of parameters such as the section breadth and depth; the area of steel; and the strengths of the concrete and steel. Where a section is subject to both an axial force and bending moments about one or both axes, interaction diagrams are commonly used to determine the area of steel required to resist the moments and forces to which the section is subjected. Structural design codes (e.g. Eurocode 2 [1]) often provide interaction diagrams for use with typical concrete sections at ambient temperature that allow the user to circumvent the cumbersome calculations necessary to determine suitable section parameters directly. However, there are many situations, such as fire loading, where structural engineers may need interaction diagrams for sections which are not covered by the standard cases. In these circumstances it is necessary to produce interaction diagrams from first principles.

This paper considers the creation, use and reliability of interaction diagrams for concrete sections; in particular those subject to fire loading. One of the problems frequently encountered in structural fire engineering is that of the definition of failure. The motivation for creating interaction diagrams for sections at high temperature lies in the difficulty of defining failure for a single element. Often failure of heated sections is loosely defined as the beginning of run-away deflections, or the time at which steel reinforcement reaches a pre-determined temperature, but such definitions are too vague to lead to efficient design. The use of carefully prepared interaction diagrams allows for a much tighter definition of section failure. Although a full understanding of the failure process requires global structural modelling, the use of an interaction diagram in conjunction with knowledge of the loading state of a section allows an engineer to determine how close a single member is to failure.

The first section of the paper reviews the available methods for constructing interaction diagrams for use in the design and also the assessment of concrete sections. It highlights the difficulties associated with obtaining interaction diagrams for determining ultimate capacity using existing methods. A new method of creating two- or three-dimensional interaction diagrams of sections under any temperature field based the tangent stiffness matrix of a section is then presented. It is anticipated that this technique will allow design engineers to make rapid assessments of the capacity of any concrete section when subject to arbitrary fire loading. The method can be implemented easily in any of the programming languages or mathematical analysis packages commonly available in design offices. . Finally the paper makes comparisons between using interaction diagrams constructed using this method to design heated concrete sections, and existing Eurocode design methods. It is concluded that current methods are not conservative.

\footnotetext{
${ }^{1}$ Address: BRE Centre for Fire Safety Engineering, The School of Engineering and Electronics, The University of Edinburgh, The King's Buildings, Mayfield Road, Edinburgh, EH9 3JL, Scotland. Email: A.Law@ed.ac.uk Tel: +44 (0)131650 7241, Fax: +44 (0)1316506781

${ }^{2}$ Address: BRE Centre for Fire Safety Engineering, The School of Engineering and Electronics, The University of Edinburgh, The King's Buildings, Mayfield Road, Edinburgh, EH9 3JL, Scotland. Email: M.Gillie@ed.ac.uk Tel: +44 (0)131650 7204,Fax: +44 (0)1316506781
} 


\section{CREATING INTERACTION DIAGRAMS - EXISTING METHODS}

\subsection{Interaction Diagrams for Ambient Temperature Design}

Interaction diagrams used for design are based on the assumption that there is a maximum allowable concrete compressive strain which prevents concrete crushing (Fig. 1). It is also frequently assumed that plane sections remain plane, that the tensile strength of concrete is negligible [2-4] and that the concrete stress distribution can be represented by a rectangular stress-block [5, 6]. From these assumptions, the derivation of an interaction diagram is relatively simple. By holding extreme fibre strains at the maximum permissible value while curvature is varied, moment and axial force (M-N) pairs that lie on the interaction diagram for the section can be obtained by appropriate integrations of the resulting stresses over the section.

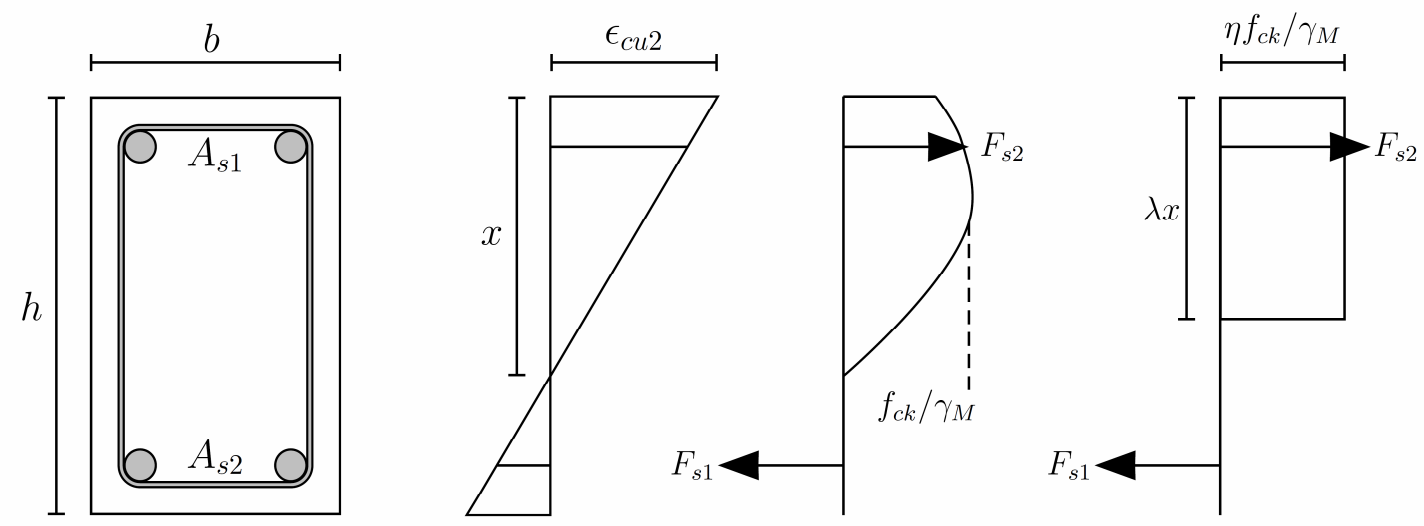

Figure 1. The development of a stress block from first principles.

This method enables the creation of interaction curves in uniaxial bending, and also interaction surfaces when biaxial bending is considered. The method is efficient because each M-N pair calculated is know to lie on the design interaction curve due to the assumption of a maximum permissible concrete strain. Diagrams produced this way can either be used directly or be normalised appropriately to allow the engineer to specify the section properties. However, because the assumptions made regarding maximum strain and the size of the associated stress block are design assumptions (which may include safety factors), the interaction diagrams obtained are not indications of failure strength, but rather show an appropriate conservative design capacity.

\subsection{Interaction Diagrams for Assessment}

Assessment of the ultimate, rather than design, capacity of a section may be required in a number of situations, including that of assessing the strength of a section under fire loading. Assessing ultimate capacity requires a different approach to that used for assessing design capacity. The assumption of a maximum fixed permissible concrete strain cannot be made because, for heated sections, the ultimate strain is temperature dependent and, consequently, the maximum moment capacity may occur at curvature that induces a strain greater than, or less than, the strain required to crush the concrete in different parts of the section. Similarly, a realistic concrete stress-strain relationship should be used, rather than a simplified stress block. Since the maximum compressive strain is not limited, it is no longer straightforward to find strain-curvature pairs that lie on the interaction curve for a section.

Several methods for obtaining interaction diagrams when maximum compressive strains are not specified have been presented. The simplest rely on variations of a "brute force" approach in which a section is analysed for many combinations of $\varepsilon_{a}$ and $\kappa$; appropriate integrations of the section stresses are then used to plot a point in M-N space for each combination. Assembly of all these points gives a "cloud" of points which are all inside or on the interaction curve [7-9]. By connecting the outer-most points in this cloud, the interaction curve can be drawn. Figure $2 \mathrm{c}$ demonstrates the generation of a number points which would contribute to the cloud of data. A significant problem with this method is that there is no clear way of determining which points lie on the interaction curve and which points lie just inside it, so determining the interaction curve from a cloud of points is awkward. These problems 
are magnified if biaxial bending is considered and an interaction surface required. The method is also computationally expensive as many integrations of the stresses over the section are required.

An alternative approach to integrating stresses over an entire section is to use the "rapid exact" inelastic biaxial bending analysis technique $[10,11]$ which allows very efficient analytical integration of the stresses. This method relies on Green's Theorem [12] to convert the costly double integration of stresses over a section to a highly efficient line integral around the section boundary. Implementation of the method requires the stress-strain relationships of the materials be analytically integrable but can be applied to any cross-section. Although computationally much more efficient than simple brute-force methods, the problem of identifying points that lie on the interaction diagram remains.

\subsection{Section Analysis at High Temperatures}

Exposure of a concrete section to a fire, and the resulting temperature increase cause the capacity to reduce and the shape of the failure surface to change. In design, several methods are available for assessment of a heated member's capacity [13]. The most commonly used and simplest is the $500^{\circ} \mathrm{C}$ isotherm method $[14,15]$. This method allows the design bending capacity of a heated section to be calculated based on the assumption that concrete retains its full strength below $500^{\circ} \mathrm{C}$ and has negligible strength above $500^{\circ} \mathrm{C}$. Reinforcement material properties are calculated based on centre-line temperatures. The design capacity is assessed using the stress block method based on accidental limitstate (fire) partial safety factors. This approach avoids the need to calculate an interaction diagram altogether but is very crude can be unconservative, as will be demonstrated later in this study.

Several authors have created M-N interaction diagrams for sections at high temperatures [7, 16, 17], and others have created moment-curvature relationships based on similar assumptions [3, 18, 19]. These studies have predominantly focussed on uniaxial bending. In order to create interaction diagrams for heated sections, it is necessary to correctly represent the degradation of material properties caused by temperature and also to represent the strains due to thermal expansion. The effects of concrete spalling on section capacity can be included by the adjustment of the geometry of the section. Once these effects have been taken into account, it is then in principle possible to create an interaction diagram using any of the (non-design) methods outlined above. However, in practice each method has practical difficulties when used at high temperature.

"Brute-force" methods are relatively simple to implement for heated sections but the problems associated with these for ambient temperature analyses are amplified for high temperature analyses as a new diagram must be generated for every cross-sectional temperature distribution. The rapid exact method becomes unwieldy at high temperatures for two reasons. First, the stress-strain relationship of concrete becomes more complex and this makes it difficult to integrate with respect to strain. Second, as the integration is over a region of both non-uniform strain and temperature, the stress-strain curve must be integrated with respect to the both change in strain and change in temperature over the region.

\section{AN ALTERNATIVE - THE TANGENT MODULUS METHOD}

In this section an alternative method is proposed to create interaction diagrams for sections both at ambient temperature and when heated by exploiting tangent stiffness matrices. The method has the key benefit of bypassing the difficulties in determining M-N pairs that lie exactly on an interaction curve or surface.

\subsection{Theory}

Although typically used in structural stability calculations as part of Shandley's tangent modulus equation [8], tangent stiffness matrices can also be used to locate failure surfaces. A section's tangent stiffness matrix relates small changes in generalized strains (typically an axial strain and two curvatures are needed for the analysis of biaxial bending of concrete sections) to small changes in the corresponding stress-resultants (an axial force and two bending moments). When a section's response is non-linear, the tangent stiffness matrix is distinct from the elastic stiffness matrix. For the set of stress-resultants mentioned, the relationship between incremental stress-resultants, tangent stiffness matrix, and incremental generalized strains of a section can be written in the standard form: 
or,

$$
\left[\begin{array}{c}
\delta P \\
\delta M_{x} \\
\delta M_{y}
\end{array}\right]=\left[\begin{array}{lll}
K_{11} & K_{12} & K_{13} \\
K_{21} & K_{22} & K_{23} \\
K_{31} & K_{32} & K_{33}
\end{array}\right]\left[\begin{array}{l}
\delta \varepsilon_{a} \\
\delta \kappa_{x} \\
\delta \kappa_{y}
\end{array}\right]
$$

where Rotter [10] gives:

$$
\delta \mathbf{F}=\mathbf{K} \delta \boldsymbol{\varepsilon},
$$

$$
\begin{aligned}
& K_{11}=\iint E_{T} d A \\
& K_{12}=K_{21}=\iint E_{T} y d A \\
& K_{13}=K_{31}=\iint E_{T} x d A \\
& K_{22}=\iint E_{T} y^{2} d A \\
& K_{23}=K_{32}=\iint E_{T} x y d A \\
& K_{33}=\iint E_{T} x^{2} d A
\end{aligned}
$$

The set of stress-resultants that lie on the failure surface of a section are those that arise when an incremental change in the generalized strain vector result in $\delta \mathbf{F}=0$. That is, stress-resultants on the failure surface are those that occur when $\mathbf{K}$ is singular, or

$$
\operatorname{det}(\mathbf{K})=0
$$

This fact can be used to determine stress-resultant vectors, $\mathbf{F}$, for a section that are located on the interaction surface if incremental strain vectors which correspond to above condition can be determined.

\subsection{Implementation}

The following calculations were all completed using the commercially available software, Matlab [20].

\section{Ambient Temperature}

Implementation of the tangent stiffness method for constructing interaction diagrams will be discussed with reference to a simple section at ambient temperature. The section dimensions and properties are shown in Fig. 2a. Assumptions made in this implementation are: plane sections remain plane; the concrete compressive stress-strain behaviour is as given in Eurocode 2 [21]; the tensile strength of concrete is zero; the stress-strain relationship of steel is elasto-plastic; and there is no bond slip between steel and concrete. Each of these assumptions has been made in various combinations by other authors $[2,9,22]$. They result in a marginally conservative estimate of strength (with the exception of bond slip). 


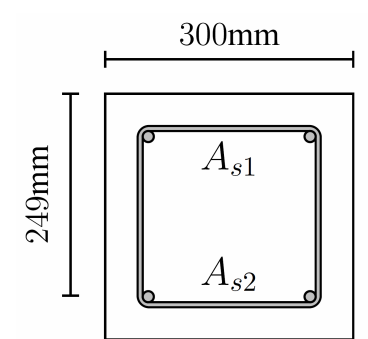

$A_{s 1}=2 \times 16 \mathrm{~mm}$ diameter

$A_{s 2}=2 \times 16 \mathrm{~mm}$ diameter

$f_{c k}=48 \mathrm{MPa}$

$f_{y}=500 \mathrm{MPa}$

a)

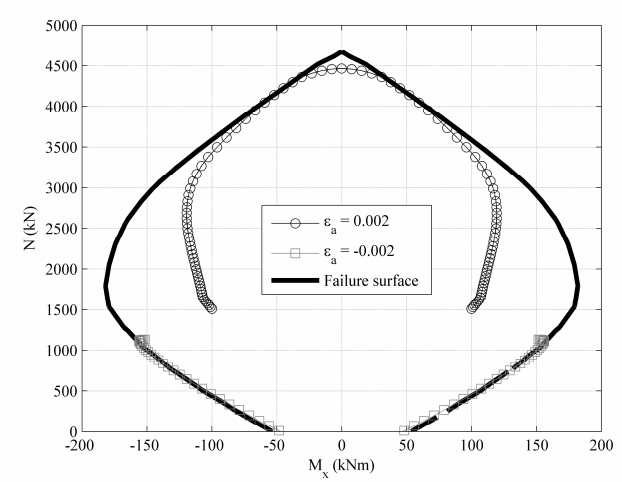

c)

Figure 2. a) Arrangement of the section, and material properties; b) Change of the determinant of the stiffness matrix with respect to curvature for two values of axial strain; c) Interaction plot for two different values of axial strain with varying curvature; d) Conceptual illustration of the search algorithm used for identifying strain-curvature points on the interaction diagram. Points where the stiffness matrix determinant is singular (circles) lie between curvatures that cause the determinant to change sign. The precise location of singularities between such curvatures is found using the secant method.

To produce the interaction curve for the section subject to uniaxial bending $\left(\kappa_{y}=0\right)$, values of curvature for which $\operatorname{det}(\mathbf{K})=0$ were found for discrete values of axial strain. These curvatures were identified using the Secant method, in conjunction with a simple search function to identify the neighbourhoods where $\operatorname{det}(\mathbf{K})$ approached zero. For example, variation of $\operatorname{det}(\mathbf{K})$ against curvature for axial strain values of $\varepsilon_{a}=-0.002$ and 0.002 is shown in Fig. $2 b$. Points where $\operatorname{det}(\mathbf{K})=0$ are marked as singularities on the figure; each of these represents a point on the interaction curve in terms of strain and curvature. Step changes in the value of $\operatorname{det}(\mathbf{K})$, caused by yielding of the reinforcement in either compression or tension, are marked by B on the figure. The corresponding curves in terms of moment and axial force are plotted in Fig. 2c. Fig. 2d illustrates conceptually an effective search algorithm for finding points where $\operatorname{det}(\mathbf{K})=0$. For incremental values of axial strain the determinant is evaluated at a number of curvatures. A change in the sign of the determinant between curvature values indicates that a region where a singularity occurs has been reached. Once the singularity region is located, the secant method can be invoked to locate the precise location of the highlighted singularities.

Once strain vectors that lay on the interaction surface had been determined, numerical integration was used to compute $\mathbf{K}$ and the corresponding $\mathbf{F}$. The final interaction curve in terms of force and moment is shown in Fig. 3 b. 


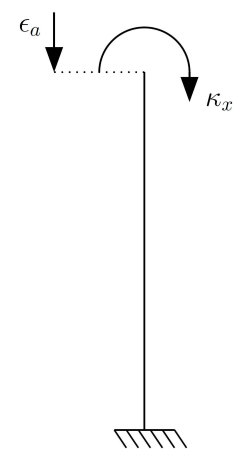

a)

Figure 3. a) Geometry, boundary conditions, and loading of the finite element model; b) Interaction diagram from tangent modulus method (bold) and the full field of data generated by the finite-element model.

The procedure was easily extended to produce interaction surfaces corresponding to biaxial bending by introducing variations to $\kappa_{y}$. This was achieved by the use of a parameter which defined $\kappa_{y}$ as a ratio of $\kappa_{x}$. Variation of this parameter from a large negative ratio to a large positive number ratio (e.g. -100.0 to 100.0) gave the full range of the biaxial failure surface, where a value of zero gave uniaxial bending about the $x$-axis. This full surface is shown in Fig. $4 \mathrm{a}$ and discussed in more detail below.

Presentation of interaction curves for uniaxial bending is well established and straightforward (e.g. Fig 2b). Presentation of interaction surfaces for biaxial bending in a form readily accessible to the design engineer is less easy. The presentation of a surface plot (Fig 4a) is not terribly useful in this respect. Visualisation choices are often dependent on the purpose for which the plot to be used. For generality, the plots in this study are presented as $M_{x}, N$ interaction diagrams with multiple curves representing different values of $M_{y}$ (Figs $4 \mathrm{~b}$ and $4 \mathrm{c}$ ). Thus, a large amount of information can be summarised on one diagram (Fig. 4d). The process employed to create these diagrams is shown sequentially in Fig 4. A single plot is sufficient for a member with a minimum of one axis of symmetry. However, where a section is entirely asymmetric, two diagrams may be required to encompass all of the information. In design, the tensile capacity of concrete sections is not normally considered. As such, just the compressive capacities are presented in this paper.

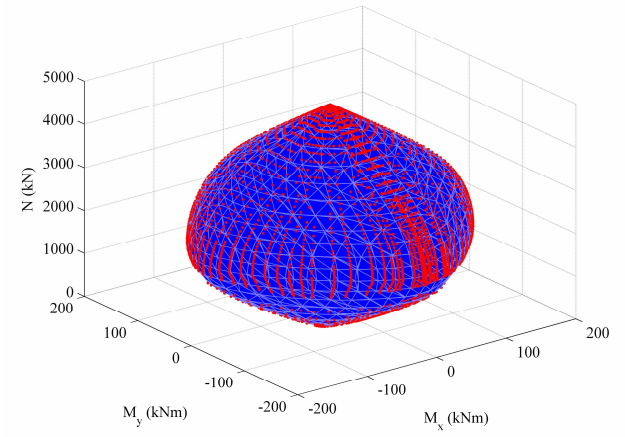

a)

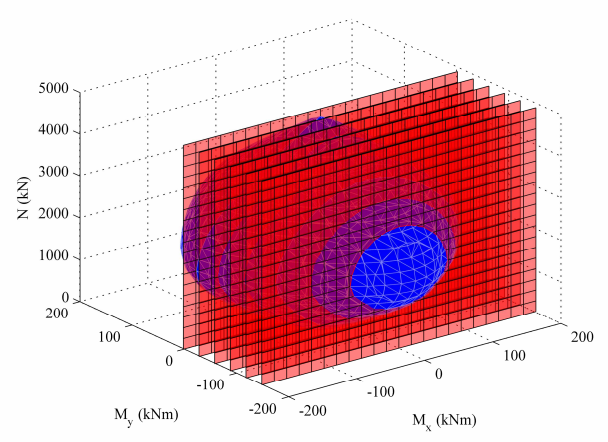

c)

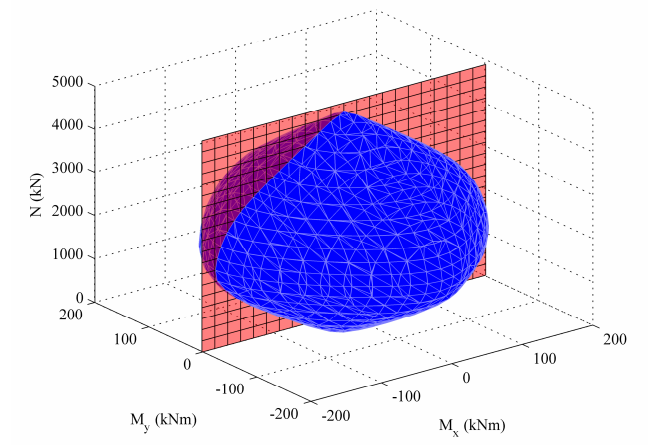

b)

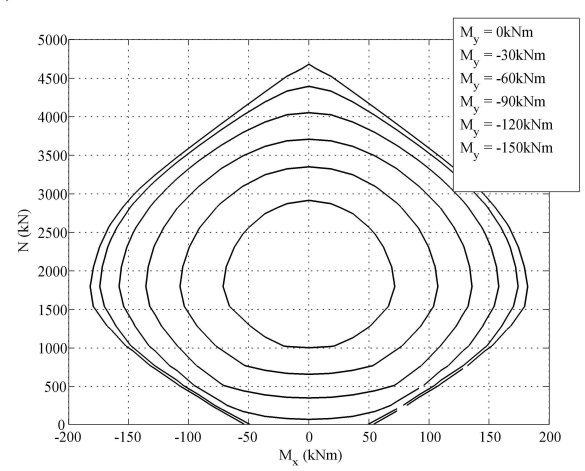

d) 
Figure 4. a) Surface plotted through all points; b) Slice taken through uniaxial bending plane; c) Multiple slices taken at different values of $M_{y}$; d) Surface removed, plot rotated and viewed from one side, and the different values of $M_{x}$ and $N$ displaced for each value of $M_{y}$.

\section{Verification}

Verification of the above implementation was undertaken by comparing the results with finite element analyses of the same problem. A stocky cross-section of the section was subject to a series of strain/curvature analyses using the finite-element program Abaqus [23] (effectively a brute-force type of analysis). The analyses were conducted on a single beam element, and the effects of non-linear geometry were neglected (to ensure a purely sectional analysis). The arrangement of the model is shown in Fig. 3a. The full "cloud" of data resulting from the finite element analyses and the failure curve resulting from employing the tangent modulus method are shown in Fig. 3b. The outer points in the data from the finite element analyses are in good agreement with the interaction curve predicted by the tangent stiffness method so the implementation is considered verified.

\section{Thermal strains}

Applying a tangent stiffness analysis to a heated section is very similar to applying it to a section at ambient temperature. However, the assumption of plane sections remaining plane must be examined more closely.

In unloaded axially and flexurally restrained members, the mechanical strain in any fibre is equal to the thermal strain. Thermal strains, therefore, directly induce stresses within the member. In unrestrained members, this is not the case; thermal strains induce changes in curvature and length, but no net moment or force [24]. Instead, they produce a deflection and internal self equilibrating internal stresses. The forces in the section balance as some regions are in compression, and some are in tension. In an unrestrained member, this leads to an initial mechanical strain state which is not zero. Thus, it has been recognised that to obtain valid load-axial strain or moment-curvature relationships, adjustments must be made to the initial state of the strain field to compensate for the thermal strain. In symmetrically heated sections, this can be done by the application of an axial strain to cancel out the axial force. The axial strain is calculated iteratively, such that the forces due to the combination of axial strain and thermal strain are in equilibrium [3].

Where a section is non-symmetrically heated, and thermal curvature is induced, the procedure is more complicated, but similar. Instead of the application of axial strain to equilibrate the axial force, a curvature and an axial strain must be applied until both the internal moment and axial force are in equilibrium. Where biaxial moments are introduced, equilibrium must be obtained by the adjustment of biaxial curvatures and axial strains.

Application of the initial strain state and subsequent calculation of the sectional yield surface allows the surface to be drawn for a cross section without reference to the member boundary conditions. In the case where an axial member remains un-restrained, no axial force will develop, and the loading state can be plotted from the applied bending moments. On the other hand, where a member is fully restrained the axial loading the member undergoes can be derived from simple calculations, or a finite element model. In this situation, the axial load would be equal and opposite to the axial load due to the initial strain state [25]. Likewise, in a flexurally unrestrained member no net bending moment would be induced by the thermal curvature. In the case where a member is at least partially restrained in rotation at both ends, bending moments would develop due to the thermal strains.

\section{Heating}

The section described and analysed above was now assumed to be uniformly exposed to a gas temperature of $600^{\circ} \mathrm{C}$ for one hour. A heat-transfer analysis was conducted using material properties from Eurocode 2 for calcareous concrete as inputs to the finite-element program Abaqus. The temperature distribution in the column at one hour is shown in Fig. 5a. Subsequently, mechanical analysis using the tangent stiffness method was undertaken to determine the interaction curve after one hour of heating. The steel stiffness and strength degradation rates given in Eurocode 2 were approximated to those of a perfectly elasto-plastic material and used in this analysis. A direct comparison between the uniaixal capacities of the ambient and heated sections can be made; Fig. 5b 
shows the progressive change in the extent of the failure surface for the value of $\mathrm{M}_{\mathrm{y}}=0$ and demonstrates the use of the tangent-stiffness method for determining interaction curves of heated sections.

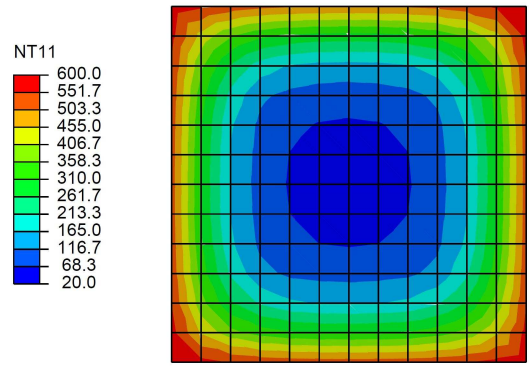

a)

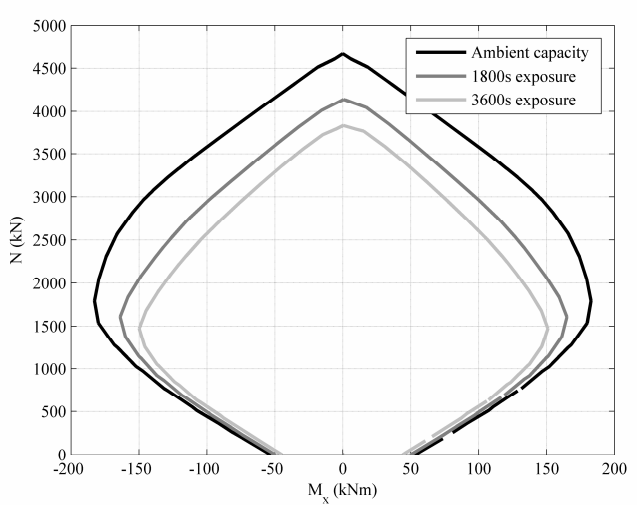

b)

Figure 5. a) Temperature distribution in the column at one hour $\left({ }^{\circ} \mathrm{C}\right)$; b) Uniaxial interaction diagram at ambient, $t=1800 \mathrm{~s}$ and $t=3600 \mathrm{~s}$.

\section{APPLICATION OF THE METHOD}

\subsection{Analysis of a Heated Section}

To show the versatility of the tangent modulus method and to compare its predictions with existing methods, a beam similar to that of Dwaikat [18] was analysed (Fig. 6a). The section was assumed to be subject to a standard fire [13] from three sides for a duration of two hours, and a heat transfer analysis of the section was conducted using Abaqus in the same manner as described above. The beam crosssection was then analysed using the tangent stiffness method for the predicted temperature fields at several intervals during the heating for a range of biaxial bending conditions. A bending capacity analysis of the beam was also conducted using the Eurocode $500^{\circ} \mathrm{C}$ isotherm method. The same heat transfer data was used for this analysis, and values of $\eta=1.0, \gamma_{M, f i}=1.0$ and $\lambda=0.8$ (Fig. 1) were used in accordance with EC2. The results of all these analyses are also shown in Fig. 6 and a direct comparison between failure surfaces at different temperatures with $\mathbf{M}_{\mathrm{y}}=0$ is shown in Fig. 7. Unlike a uniformly heated section [17, 26], an asymmetrically heated section's interaction surface distorts as well shrinking during heating [16]. The asymmetry in the failure diagram results from the reference axis remaining at a constant location while the plastic neutral axis moves through the section.

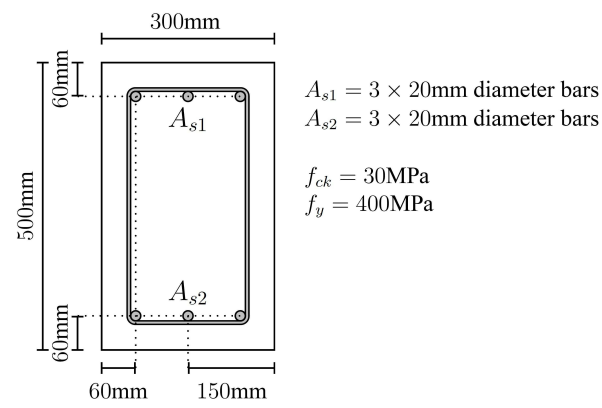

a)

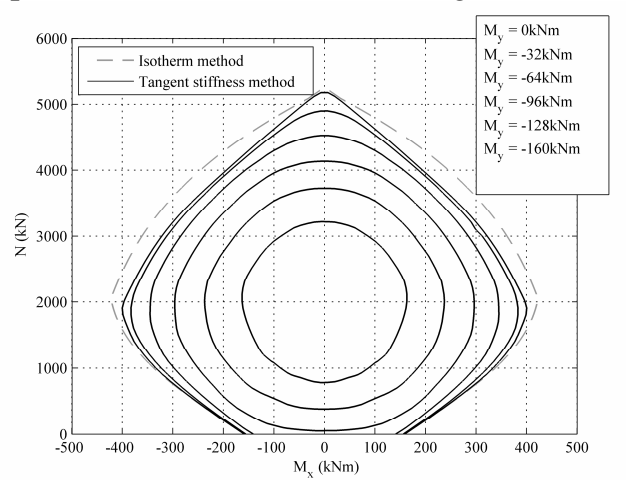

b 

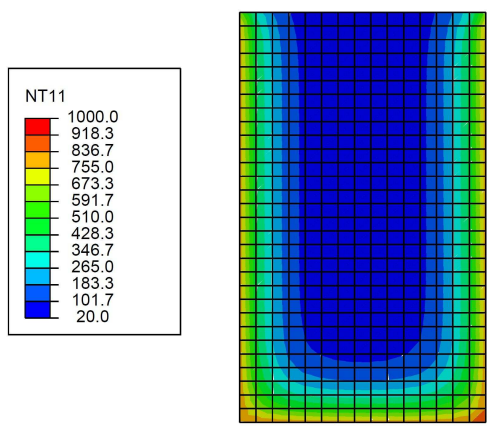

c)

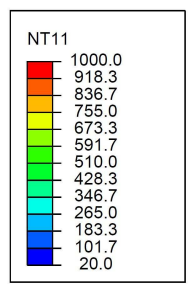

e)

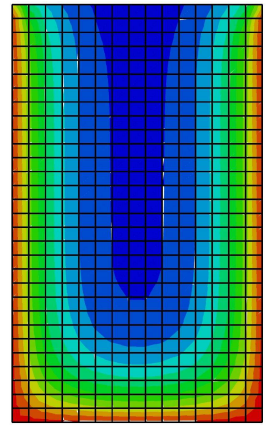

Figure 6. a) Arrangement of the section; b) Ambient interaction diagram for the section using both tangent method and isotherm method; c) Section temperature profile after one hour $\left({ }^{\circ} \mathrm{C}\right) ; d$ ) Interaction diagram for the section at one hour using both tangent method and isotherm method; e) Section temperature profile after two hours $\left({ }^{\circ} \mathrm{C}\right)$; f) Interaction diagram for the section at two hours using both tangent method and isotherm method.

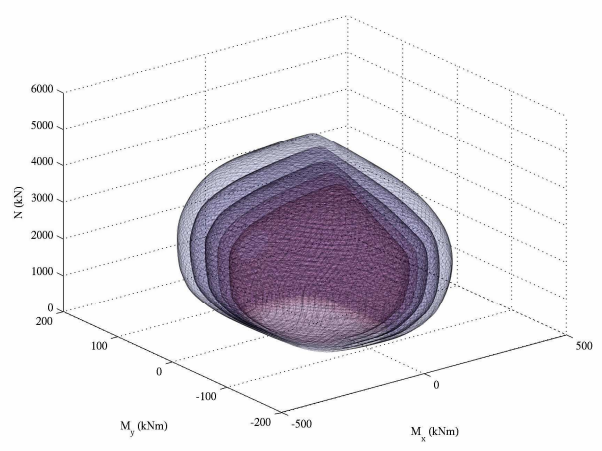

a)

Figure 7. a) Multiple failure surfaces; b) Comparison of uniaxial interaction diagram

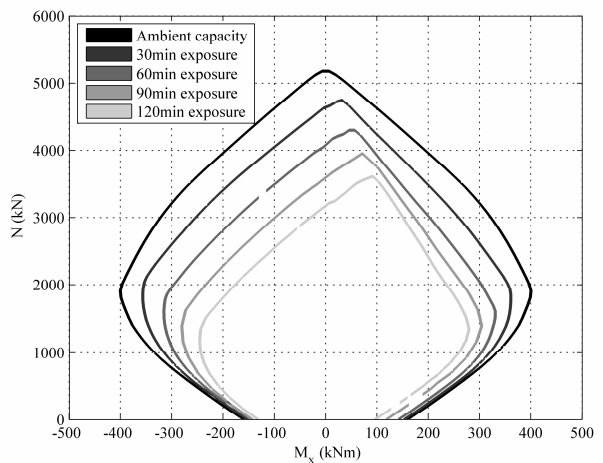

b)

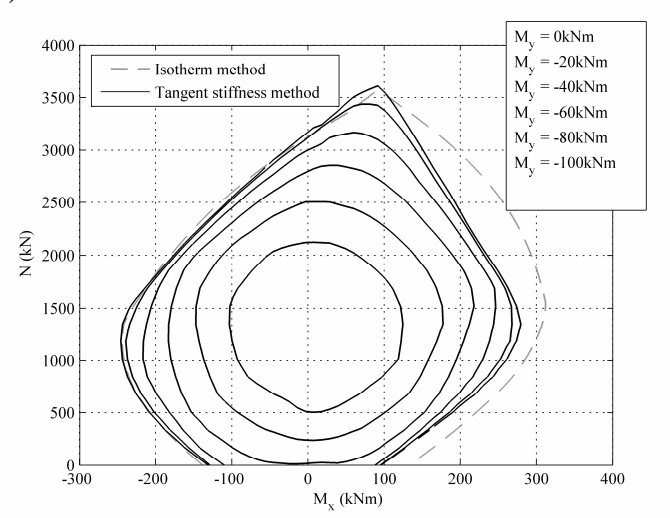

f)

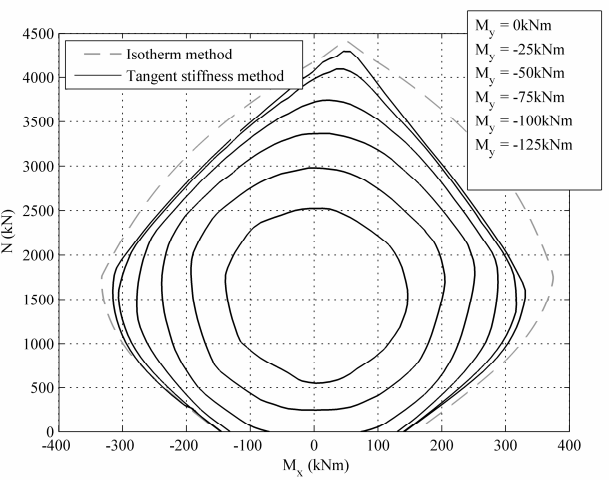

d) 
particularly the case in sagging, where up to a $35 \%$ overestimation is obtained. Pure hogging produces a moment overestimation of $5 \%$.

- The isotherm method almost universally over-estimates the capacity of the section under combinations of sagging moment and axial force. In the high axial load region, this overestimation grows from $5 \%$ to $15 \%$ as the section is heated.

- In contrast, overestimation of the hogging moment decreases as the section is heated. In fact, when the section is at its hottest, the agreement between the two results is very good with an error of less than $3 \%$ throughout.

These observations of the uniaxial bending results for both methods show that the $500^{\circ} \mathrm{C}$ isotherm method cannot be assumed to be a conservative design approach. It consistently overestimates the capacity of the section, particularly in sagging. Other researchers have found the isotherm method to be unconservative in pure uniaxial bending [27]. This study shows that the unconservatism extends to bending with different levels of axial force.

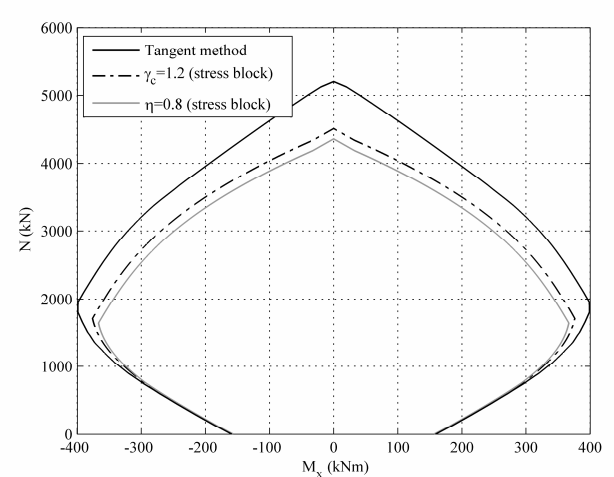

a)

Figure 8. a) Different individual safety factors; b) Equivalent capacities for the tangent method, stress block method, full stress-strain curve method, design safety factors, and with a reduced stress block.

Although it is unsurprising that a crude assessment such as the isotherm method does not give accurate results, it is of concern that the results are unconservative. The causes of this unconservatism are twofold: the specific differences between the prediction of sagging and hogging capacity at higher temperatures are largely attributable to the assumptions inherent in the isotherm method. However, it is possible to attribute some of the error directly to the use of a stress block technique.

Heated concrete reaches its maximum compressive strength at a higher strain than cold concrete. Thus, in order for a section to develop a given axial load when subject to sagging bending, the neutral axis will be lower in a heated section than a cold section. Consequently, the internal lever arm and hence the moment capacity will be reduced. These effects are fully captured the tangent stiffness method but ignored in the isotherm method, which is unconservative as a result. In hogging, however, the effect described is largely cancelled out by the fact that the heated section soffit has been effectively removed in the isotherm method due to its high temperature. The removal of this area and its corresponding contribution to the total bending moment largely cancels out the overestimation caused by the inappropriate maximum strain assumptions. The above considerations highlight how the isotherm method has certain assumptions that inevitably will lead to unconservative predictions for some straincurvature combinations, as demonstrated above. However, there are aspects of the underlying stressblock method that produce further inaccuracies.

The dimensions of the stress block used in this method are determined by the parameters $\lambda$ and $\eta$ as shown in Fig. 1. These in turn are specified to ensure that under pure bending at ambient temperature the stress-block applies a force of the same magnitude and with the same lever-arm about the neutral axis as would occur if a full material stress-strain curve were used in an analysis. For a concrete strength of 50MPa or less, values of $\lambda$ and $\eta$ are given as 0.8 , and 1.0 respectively in Eurocode 2. Under accidental loading (fire) conditions, the value of $\gamma_{M}$ for concrete in Eurocode 2 is modified from 1.5 to 1.0 thus meaning any section analysis undertaken using the isotherm method has effectively no material safety factor and relies only on the values of $\lambda$ and $\eta$. These simplified terms cannot accurately represent both the correct stresses and the appropriate lever arms which result from the true concrete stress-strain curve under any but the simplest load cases. This is particularly the case when the neutral axis is not in the section, and the stress block acts over the whole section. Although an accurate prediction of the pure axial capacity can be obtained (because $\eta=1$, and the value of $\lambda$ is irrelevant) 
this study shows that this method produces inaccurate and unconservative results when bending moments are present.

Given the interdependence of these safety factors for obtaining a conservative design at high temperature, it is of interest to examine the effect of arbitrary modifications of the stress-block. Modification of either $\eta$ to 0.8 , or $\gamma_{c}$ to 1.2 , allow a conservative result to be obtained for the ambient case (Figure 8a). However, this is an inefficient approach as in both of these cases the maximum axial capacity is significantly underestimated. Furthermore, neither of these modifications is sufficient to ensure that the entire interaction envelope of the isotherm method was within the failure surface given by the tangent stiffness method for the heated $(120 \mathrm{~min})$ case. Further modification of the material partial safety factors to the ambient temperature design values (1.15 and 1.5 for steel and concrete respectively) in the heated case reveals that the predictions of moment capacity remain unconservative (Figure 8b). The overestimation caused by the stress block technique can be further analysed by applying the isotherm technique with a full concrete stress-strain relationship Fig. 8b. This modification, however, is not sufficient to allow for a fully conservative capacity assessment.

The variation of parameters and techniques in the manner described above allows the source of the unconservatism in the heated sections to be more closely analysed. It is possible to obtain a more conservative result by modification of the stress block. However, despite modification of the partial safety factors, the permissible sagging moment in the heated section (as predicted by the isotherm method) remained significantly unconservative. The sagging overestimation is specifically caused by the difference in the location of the neutral axis between the two methods. The lower neutral axis in the heated section causes a lower moment to be generated than in the ambient, reduced area, section. The adoption of a more realistic stress-strain curve for the ambient concrete, does not give a conservative result. It is therefore concluded that the unconservatism in the predicted capacities is caused more by the assumption of ambient concrete behaviour inherent in the isotherm method, rather than because of the use of the stress block technique. Another cut-off value such as $400^{\circ} \mathrm{C}$ or $300^{\circ} \mathrm{C}$ would, inevitably, give a more conservative answer. However, this would not solve the underlying problem with the cool concrete assumption.

\section{5) CONCLUSIONS}

Several conclusions can be drawn from this study:

- The failure surfaces of ambient and heated reinforced concrete sections can be found rapidly and accurately by locating singularities in the sectional tangent modulus matrix.

- The $500^{\circ} \mathrm{C}$ isotherm method is unconservative in predicting the failure surface of a heated section due to the stress block method (see above) and the assumption of uniform temperature in the concrete.

- Biaxial failure surfaces for ambient and heated reinforced concrete sections can be clearly represented on a single diagram.

- When all the partial safety factors are removed, the stress block method is unconservative in predicting the failure surface of a section at ambient temperature.

\section{ACKNOWLEDGEMENTS}

The authors of this paper would like to acknowledge BRE Trust for their financial support of this project. The authors would also like to thank Professor J. Michael Rotter and Dr Antonis Giannopoulos for their help in developing this work.

\section{REFERENCES}

[1] EN1992-1-1. Eurocode 2: Design of Concrete Structures - Part 1-1: General rules and rules for buildings, 1999.

[2] Yen JYR. Quasi-Newton Method for Reinforced-Concrete Column Analysis and Design. Journal of Structural Engineering 1991;117:657.

[3] El-Fitiany SF, Youssef MA. Assessing the flexural and axial behaviour of reinforced concrete members at elevated temperatures using sectional analysis. Fire Safety Journal 2009;44:691.

[4] Rocca S, Galati N, Nanni A. Interaction diagram methodology for design of FRP-confined reinforced concrete columns. Construction and Building Materials 2009;23:1508. 
[5] Mosley B, Bungey J, Hulse R. Reinforced Concrete Design to Eurocode 2. New York: Palgrave MacMillan, 2007.

[6] Nawy EG. Reinforced Concrete; a Fundamental Approach. London: Pearson, 2009.

[7] Gillie M, Usmani A, Rotter M. Bending and Membrane Action in Concrete Slabs. Fire and Materials 2004;28:139.

[8] Bazant ZP, Cedolin L. Stability of Structures: Elastic, Inelastic, Fracture, and Damage Theories. New York: Oxford University Press, 1991.

[9] Rodriguez JA, Aristizabal-Ochoa JD. Biaxial Interaction Diagrams for Short RC Columns of Any Cross Section. Journal of Structural Engineering 1999;125:672.

[10] Rotter M. Rapid Exact Inelastic Biaxial Bending Analysis. Journal of Structural Engineering 1985;111:2659.

[11] Fafitis A. Interaction Surfaces of Reinforced-Concrete Sections in Biaxial Bending. Journal of Structural Engineering 2001;127:840.

[12] Kreyszig E. Advance Engineering Mathematics. New York: Wiley, 1999.

[13] EN1991-1-2. Eurocode 1: Actions of Structures - Part 1-2: General Actions - Actions on Structures Exposed to Fire, 1999.

[14] EN1992-1-2. Design of Concrete Structures - Part1-2: General rules- Structural fire design, 1992.

[15] Lennon T, Rupasinghe R, Canisius G, Waleed N, Matthews S. Concrete Structures in Fire: Performance, design and analysis. Watford: BRE press, 2007.

[16] Garlock MEM, Quiel SE. Plastic Axial Load and Moment Interaction Curves for FireExposed Steel Sections with Thermal Gradients. Journal of Structural Engineering 2008;134:874.

[17] Bamonte P. On the Role of Second-Order Effects in HSC Columns Exposed to Fire. In: Tan KH, Kodur VKR, Tan TH, editors. Fifth International Conference of Structures in Fire. Singapore, 2008.

[18] Dwaikat MB, Kodur VKR. A numerical approach for modeling the fire induced restraint effects in reinforced concrete beams. Fire Safety Journal 2008;43:291.

[19] Rodriguez-Gutierrez JA, Aristizabal-Ochoa JD. Reinforced, Partially, and Fully Prestressed Slender Concrete Columns under Biaxial Bending and Axial Load. Journal of Structural Engineering 2001;127:774.

[20] MATLAB. Natick: The MathWorks Inc, 2008.

[21] EN1992-1-2. Design of Concrete Structures - Part1-2: General rules- Structural fire design, , 1992.

[22] Bonet JL, Miguel PF, Fernandez MA, Romero ML. Analytical Approach to Failure Surfaces in Reinforced Concrete Sections Subjected to Axial Loads and Biaxial Bending. Journal of Structural Engineering 2004;130:2006.

[23] Abaqus. Abaqus Analysis User's Manual. Providence: Dassault Systemes Simulia Corp, 2008.

[24] Tassios TP, Chronopoulos MP. Structural Response of RC Elements Under Fire. The Structural Engineer 1991;69:227.

[25] Usmani AS, Rotter JM, Lamont A, Sanad AM, Gillie M. Fundamental Principles of Structural Behaviour under Thermal Effects. 36 2001:721.

[26] Taerwe L. From Member Design to Global Structural Behaviour. In: Rodrigues JPC, Khoury GA, Hoj NP, editors. International Workshop of Fire Design of Concrete Structures. Coimbra, Portugal, 2007. p.253.

[27] Goncalves MC, Rodrigues JPC. Ultimate Bending Moment Capacity of Reinforced Concrete Beam Sections at High Temperatures. In: Rodrigues JPC, Khoury GA, Hoj NP, editors. International Workshop of Fire Design of Concrete Structures. Coimbra, Portugal, 2007. p.281. 
Open access copy. For published version see: http://dx.doi.org/10.1016/j.engstruct.2010.02.012 
Open access copy. For published version see: http://dx.doi.org/10.1016/j.engstruct.2010.02.012 
Open access copy. For published version see: http://dx.doi.org/10.1016/j.engstruct.2010.02.012 